\title{
Optimization Design of Multi-material Micropump Using Finite Element Method
}

\author{
Meiling Zhu ${ }^{\mathrm{a}, *}$, Paul Kirby, Martin Wacklerle ${ }^{\mathrm{b}}$, Markus Herz ${ }^{\mathrm{b}}$, Martin Richter ${ }^{\mathrm{b}}$ \\ ${ }^{a}$ Cranfield University, Cranfield, Bedfordshire, UK \\ ${ }^{b}$ Fraunhofer Institute for Reliability and Microintergration (IZM), Munich, Germany

\begin{abstract}
*Corresponding author's address: Building 70, Department of Materials, School of Applied Sciences, Cranfield University, Cranfield, Bedfordshire, MK43 0AL, UK. Tel.: +44 (01234) 750111 ext. 2092; Fax: +44 (01234) 751346; e-mail address: m.zhu@cranfield.ac.uk.
\end{abstract}

\begin{abstract}
This paper presents a micropump fabricated from low-cost materials with specific goal of cost reduction. The micropump does not require any valve flap and comprises of one plastic pump polyether-ether-ketone (PEEK) body, one metal diaphragm, and three piezoelectric ceramics to form piezoelectrically actuated diaphragm valves. The valve actuation simplifies micropump structural designs and assembly processes to make the pump attractive for low cost bio-medical drug delivery applications. A detailed optimization design of geometric parameters of the piezoelectrically actuated diaphragm is undertaken by use of 3D finite element method (FEM) to maximize piezoelectric actuation capability and ensure actuation reliability. An optimized geometric dimensional design: the ratio of thicknesses between the piezoelectric ceramics and the metal diaphragm, and the lateral dimension of the piezoelectric ceramic, is obtained through simulations. Based on the optimized design, a good agreement has been reached between simulated and measured strokes of the micropumps. The tested results show that the micropump has a high pump flow rate for air, up to $39 \mathrm{ml} / \mathrm{min}$, and for water up to $1.8 \mathrm{ml} / \mathrm{min}$, and is capable of ensuring diaphragm's maximum stress and strain is within material strength for reliable work.
\end{abstract}

Keywords: Micropump, plastics, finite element analysis, piezoelectric ceramics, piezoelectrically actuated diaphragm. 


\section{Introduction}

Micro total analysis system or $\mu$ TAS are becoming increasing important for large variety applications, such as pharmaceutical and bio-medical drug delivery systems, since the concept was introduced in 1990 [1] and [2]. The earliest development of micropump was in 1980 with a peristaltic micropump using piezoelectric biomorphies [3]. The earliest silicon micropump, that uses sputtering of the piezoelectric films directly onto the silicon pump diaphragm, was presented in 1988 [4]. Since then, many other integrated silicon based micropumps, mostly using piezoelectric actuation, were presented [5, 6]. Silicon MEMS micropumps have found several commercial applications for insulin delivery, in gas control as fluidic valves and in inject technology [7-8]. Reyes et al. [9] provides a comprehensive overview of the important developments in technology, theoretical understanding and applications in the field. During the developments, a continuous challenge has been the transport and pumping of small quantities of biological fluids on the order of a few micro/mill-litres per minutes.

However, material cost of silicon and elaborate micro-manufacturing processes burden the use of such silicon micropumps because the micropumps require expensive silicon micromachining facilities and processes. The use of plastics has already been shown to have great potential to reduce the fabrication cost. This is due to the fact that plastics are disposable, low cost, and offer a competitive manufacturing process in mass production [10][12]. To date, several of micropumps have been developed. Examples include ThinXXs [13], Bartels Mikrotechnik [14], and Star Micronics Companies [15] use Cyclo-Olefin-Copolymers or Polyimide as the pump body material. Nevertheless, the pumps have passive micro check valves and require advanced micro-manufacturing processes and specialized assembly techniques. Richter et al. [16] have recently developed a plastic micropump which comprises five parts: one plastic pump body, one metal diaphragm, and three piezoelectric ceramics. This micropump overcomes the passive micro check valve drawback by having a simple 
valve actuation design: piezoelectrically actuated diaphragm valves without requiring any valve flap. The use of plastic material in the simplified structural design, fabrication and assembly processes, and makes the pump attractive for low cost applications. While the micropump has been presented before, its optimization design has not been done yet.

In this paper, therefore, we focus on optimization of the piezoelectrically actuated diaphragm for actuation capability and reliability of the micropump. This is mainly due to the fact that the higher the applied voltage, the higher the deflection of diaphragm and thus the higher the pump flow rate. But a higher applied voltage will lead to a higher stress and a higher strain in the piezoelectric ceramics and possibly makes the pump unreliable to operate if the stress and strain is beyond material strength due to brittle nature of piezoelectric ceramics. In order to make sure of the pump with high pumping rate and high reliability, there is a need to predict the pump actuation capability and reliability and optimize geometric dimensional design of the piezoelectric diaphragm. The results presented consist of work previously reported in a proceeding paper [17] with comparisons of computational and experimental results.

\section{Description of the micro-pump}

A multi-material micropump studied here is shown in Fig.1 in an exploded view. It is comprised of solely five functional parts: one plastic body, one metal diaphragm, and three piezoelectric ceramics. Three piezoelectric ceramics are glued onto the metal diaphragm to form three actuation units to control diaphragm valve opening and closing for generation of pumping motion. The metal diaphragm is bonded to the plastic body to form two valve seats on the left and right, and one pump chamber sit in the centre.

A photo of plastic micropump body is shown in Fig. 2. An interconnection channel with a slot shape between valve seats and micro-pump chamber are designed to allow the flow through fluidic chambers and so as to reduce the flow resistance. A capillary stop channel is also 
designed around the plastic body to prevent glue to flow into the chambers after bonding the metal diaphragm to the plastic body. Because there are burrs at the edges after dicing of the metal diaphragm, a burr channel was also designed to absorb the burr of the metal diaphragm after assembly. The electrical connections to the top and bottom of piezoelectric ceramics were done by wire bonding to a standard connector.

The operational principle of the pump is described as follows. When a proper voltage is applied to one of the piezoelectric ceramics, there will be a downward deflection in the metal diaphragm. For example, the downward deflection, shown in Fig. 3, will close the left orifice in the plastic body and the valve will be closed if the piezoelectric ceramics is able to excite an enough deflection in the metal diaphragm. When the applied voltage is zero, there will be no downward deflection in the metal diaphragm and the valve will be opened. Furthermore, when there are three alternative electrical voltages with given phase shifts, shown in Fig. 3, independently applied to the piezoelectric ceramics, the valves will be opened and closed with a proper sequence and then pumping mode will be generated. As the phase shifts among three electrical signals are adjustable, the fluid can be pumped from the left seat to the right seat, and vice versa. So the pump is capable of pumping bidirectional. It is worthwhile to mention that a negative voltage is also applied to the designed micro-pumps because the negative voltage has an advantage of exciting an upward deflection to reduce the fluidic resistance of the valves and increase the pump volume.

In order for the pump to handle and dose chemical gases and liquids, Polyether-etherketone (PEEK) of plastics was chosen as material for the pump body because PEEK has the property of very good stability and resistance to a broad range of chemical gases and liquids. It should be mentioned that PEEK is an expensive material but only a small amount of PEEK (a few grams) is needed for the micropump and so a low cost micropump can still be achieved. It should be noted that for a true low cost it is advisable to use a different material 
rather than PEEK and a further investigation of the pump body material is needed. However, this issue is outside the scope of the current paper. Stainless steel (no.1.4310, from Ergeste) was chosen as material for the metal diaphragm. Adhesives for bonding between the metal diaphragm and the plastic body have been tested and special methanol stable epoxy glue is utilised. Since the glue is stable to methanol solvents, the pump is capable for fuel cell applications. In addition, to improve the sealing of the interface and prevent the leakage of the pump, a layer of polydimethylsiloxane (PDMS) elastomer (PDMS Sylgard, Dow Chemicals) was deposited to the metal diaphragm by spin coating with a two step process: the first step with $1500 \mathrm{r} / \mathrm{min}$ and the second step with $6000 \mathrm{r} / \mathrm{min}$. The ratio between binder and hardener was chosen to be 9:1. The layer was hardened for $5 \mathrm{~min}$ on a hot plate. These layers had a thickness of $6 \mu \mathrm{m}$.

\section{Optimization of the piezoelectric diaphragm}

As general rule the higher the applied voltage, the higher the deflection of diaphragm and the higher the pump flow rate. Unfortunately, a higher deflection leads to a higher stress and a higher strain in the piezoelectric ceramics and further leads to the piezoelectric actuator reliability deficiency if the stress and strain is beyond material strength due to brittle nature of piezoelectric ceramics. In order to ensure the pump work reliably, this section will study the micropump actuation capability and reliability and then based on the study, obtain an optimized dimensional design for the piezoelectric diaphragm.

\subsection{FEA Method}

Commercially available finite element analysis (FEA) software (ANSYS Inc. Canonsburg, PA), was utilized to calculate actuation capability and reliability of piezoelectrically actuated diaphragm. Here the actuation capability means how much deflection piezoelectric ceramics can excite in the diaphragm and the reliability means that the maximum stress and strain 
generated in the metal diaphragm and the piezoelectric ceramics should be below material strength limits. So the deflection of the diaphragm, below called stroke $(S)$, and the maximum stress in metal diaphragm $\left(\sigma_{\max , m}\right)$ and the maximum stress and strain in piezoelectric ceramics $\left(\sigma_{\max , p} / \varepsilon_{\max , p}\right)$ were chosen as the criteria to measure the actuation capability and reliability of the micropump as described below. The reason for this is that the stroke and stress are the key performance parameters for optimized structural design with a higher pump flow rate and for ensuring the maximum stress and strain within material strength.

The geometric parameters of modelled piezoelectric diaphragm are shown in Fig. 5. The metal diaphragm in-plane dimensions were designed to be fixed at $44 \mathrm{~mm} \times 16.95 \mathrm{~mm}$ with the thickness of $0.05 \mathrm{~mm}$, and the valve chamber sizes were fixed at $14 \mathrm{~mm}(I) \times 14 \mathrm{~mm}(I)$ with radius fillet of $4.8 \mathrm{~mm}$ at the corners, where $l$ represents the lateral dimension of the square valve seat, in-plane dimensions of the valve chamber sizes and pump chamber are designed based on the designed flow rate, and the depth are designed to adapt to strokes actuated by piezoelectric ceramics. Piezoelectric ceramic structural parameters, $l_{p} \times l_{p}$ and $t_{p} / t$, were chosen to be variable, where $l_{p}$ and $t_{p}$ represent the length and thickness of square piezoelectric ceramics, respectively, $t$ is the thickness of metal diaphragm and so $t_{p} / t$ is the ratio of the thicknesses. It should be noticed that $l_{p}<l$ due to structural limitation. As the glue is very thin, the thickness of glue is ignored in all the simulations.

The developed FEM model of piezoelectric diaphragm is shown in Fig. 6 with meshes. The model uses the 8-node, hexahedral, coupled-field element SOLID5 for the piezoelectric materials and the 8-node, linear, structural element SOLID45 for the non-piezoelectric materials. The material parameters and geometrical dimensions used in the model are listed in Table 1 except where specifically mentioned in the text. Static analyses were performed to determine the stroke, the maximum stress in the metal diaphragm and the maximum 
stress/strain in the piezoelectric ceramics.

\subsection{FEA results and analyses}

\section{A. Distribution of simulated deflection}

Fig. 7 shows the distribution of simulated deflection along the metal diaphragm length direction when a voltage of 100 volts is applied to the left, centre, and right piezoelectric ceramics, respectively. It can be seen that a large stroke, about $62 \mu \mathrm{m}$, is achieved in all three chambers for the designed micro-pump with the dimensions listed in Table 1. As a large stroke leads to a high pumping rate, the micropump has a potential of pumping high volume of fluids and gases.

\section{B $S$ and $\sigma_{\max , m}$ as functions of $V_{a}$}

Fig. 8 shows $S$, which occurs at the centre of diaphragm, and $\sigma_{\max , m}$, which occurs at the four corners of piezoelectric ceramics in the metal diaphragm, as functions of $V_{a}$. From Fig. 8 , it can be seen that the higher $V_{a}$, the higher $S$ and the higher $\sigma_{\max , m}$. It seems that there is a linear relationship between $S$ and $V_{a}$, and between $S$ and $\sigma_{\max , m}$. However, it should be borne in mind that there is a non-linearity in reality when the applied voltage is larger than 100 volts. The reason for leading to this conclusion is that a linear piezoelectric theory is used in the simulation

\section{C. $S$ and $\sigma_{\max , m}$ as functions of $l_{p}$}

Fig. 9 shows $S$ and $\sigma_{\max , m}$ as a function of the piezoelectric ceramics length $l_{p}$. From Fig. 9 , it can be observed that when $l_{p}$ increases but $l_{p}<9.6 \mathrm{~mm}, S$ and $\sigma_{\max , m}$ increase but when $l_{p}>9.6 \mathrm{~mm}, S$ starts to decrease and $\sigma_{\text {max }, m}$ starts to increase sharply. So there exists an optimized piezoelectric ceramics length $l_{p}$ for the optimized design, which is $9.6 \mathrm{~mm}$ and means that the pump designed at this length has a potential of achieving a higher stroke and not suffering higher maximum stress when the chamber size is designed at $14 \mathrm{~mm} \times 14 \mathrm{~mm}$ with 
radius fillet of $4.8 \mathrm{~mm}$.

D. $S$ and $\sigma_{\max , m}$ as functions of $t_{p} / t$

Fig. 10 shows $S$ and $\sigma_{\max , m}$ as a function of $t_{p} / t$, where $t$ is fixed at $0.05 \mathrm{~mm}$. For a comparative study reason, the electric field strength applied to the piezoelectric ceramics was fixed at 1000 volts $/ \mathrm{mm}$ for all the simulations and this leads to the relationship that the applied voltage is proportional to $t_{p} / t$. From Fig. 10, it can be seen that when $t_{p} / t=1.4$, that is, $t_{p}=70 \mu \mathrm{m}, S$ reaches a maximum value, about $67 \mu \mathrm{m}$; when $t_{p} / t>1.4, S$ starts to decrease while $\sigma_{\text {max }, m}$ continues rising until $t_{p} / t=2.4$, that is, $t_{p}=120 \mu \mathrm{m}$, and then $\sigma_{\max , m}$ almost keeps a level, about $166 \mathrm{MPa}$ when $t_{p} / t=2.4$. Theoretically, an optimized $t_{p} / t$ for designed micro-pump should be taken to be 1.4 , that is, $t_{p}=70 \mu \mathrm{m}$, as this value could make the pump have the largest stroke and suffer the highest stress. But due to difficulties in fabrication of such thin bulk piezoelectric ceramics and the availability of minimum thickness of piezoelectric ceramics is about $100 \mu \mathrm{m}$ for the time being, $t_{p}$ is designed at $100 \mu \mathrm{m}$ for the developed and tested pump. It is worthwhile to mention that, for $0.6<t_{p} / t<1.4$, that is, 30 $\mu \mathrm{m}<t_{p}<70 \mu \mathrm{m}$, piezoelectric ceramics could be available in the thick film form although currently the available piezoelectric coefficients of the thick film are much less than those in bulk form because thick film material is a relative new and still in the development stage. If similar piezoelectric coefficients of thick film as those of bulk material are achieved, it is recommended to utilize thick film piezoelectric ceramics to design the micropump because they could excite a higher stroke. Furthermore, for $t_{p} / t<0.2$, that is, $t_{p}<10 \mu \mathrm{m}$, it can be seen that the actuated stroke is very small, about $17.5 \mu \mathrm{m}$ at $t_{p} / t=0.2$, not enough to form pumping motion for the designed pump. So it is not recommended to use thin film piezoelectric ceramic to design the micro-pump due to the small stroke. 


\section{E. $\sigma_{\max , p}$ and $\varepsilon_{\max , p}$ for piezoelectric ceramics}

Figs. 11, 12 and 13 show $\sigma_{\max , p}$ and $\varepsilon_{\text {max }, p}$ in the piezoelectric ceramics as functions of $V_{a}$, $l_{p}$ and $t_{p} / t$, respectively. From these figures, very similar conclusions can be drawn as ones from Figs. 8, 9 and 10. Because of brittle nature of piezoelectric ceramics, piezoelectric material can not be subjected to a higher tensile stress and a higher tensile strain than material strength limits. So for reliability analyses of piezoelectric ceramics, the tensile strain no more than $0.1 \%$, that is, $\varepsilon_{\max , p}<0.1 \%$, was chosen as a criterion during reliability study. Because tensile strain in piezoelectric ceramics is larger than $0.1 \%$, it will possibly cause the piezoelectric ceramics to crack So from Figs. 11, 12, 13, it can be concluded that the optimized applied voltage is about 100 volts and optimized structural parameters are $l_{p}=9.6 \mathrm{~mm}$ and $t_{p} / t=2$, that is, $t_{p}=100 \mu \mathrm{m}$, and the designed pump using such parameters can have maximum stroke and work reliably.

\section{Micropump testing}

Taking the optimized geometry parameters obtained in the simulation, three piezoelectric ceramics were glued onto the metal diaphragm. To verify the capabilities of piezoelectric actuators, the strokes of the left, centre, and right diaphragms were measured at voltages between 20 volts and 100 volts by use of the laser profilometry laser scan system (Microfocus from UBM, USA). Similar measurement results were obtained and Fig. 14 shows the strokes actuated by the central piezoelectric ceramics at the diaphragm. A very large stroke, about $55 \mu \mathrm{m}$, was achieved at the applied voltage of 100 volts. It is also observed that the higher the applied voltage, the higher the stroke when $V<100$ volts. The trend well agrees with the simulation results in Section 3, shown in Fig. 8. It should be mentioned that there is some difference between the simulated and tested results in the stroke through comparisons, shown in Fig. 8. The possible reasons are that (1) actual piezoelectric ceramic property parameters vary from the catalogue parameters and (2) the tolerances of the piezoelectric ceramic geometric dimensions possibly causes the difference.

Micropumps with and without PDMS coating were fabricated and tested. Figs. 15 and 16 
show forward and backward flow rates of the micropump with PDMS coating as function of actuation frequency, for air and water. From these figures, it can be seen that the micropump enables to pump water and air and bidirectional pumping (forward and backward pumping) and the flow rates is a function of actuation frequency. The maximum flow rates of $39 \mathrm{ml} / \mathrm{min}$ were achieved for air at the actuation frequency of about $225 \mathrm{~Hz}$ and applied voltage of 100 volts, and $1.8 \mathrm{ml} / \mathrm{min}$ for water at about $17 \mathrm{~Hz}$. As mentioned in Section 2, PDMS coating is used to improve the sealing and prevent the leakage of the micro pump at the interface. Tested results have shown a good improvement of pumping performance in flow rate as for the micropump without PDMS coating.

\section{Conclusions}

An optimisation design of the micropump, using multi-materials for low cost applications, has been presented with actuation and fluidic test results in this paper. As the micropump comprises solely five functional parts: one plastic pump body, one metal diaphragm and three piezoelectric ceramics, the micropump has a simple structure, easy to assemble and suitable for low cost applications. Furthermore, the pump body is made of Poly-Ether-Ether-Ketone (PEEK), which is chemical inertia for a broad range of liquid and gases, so it is able to work with aggressive gases and liquids.

A detailed parameter design analysis and optimization of piezoelectric diaphragm has been performed based on 3D finite element method (FEM). An optimized geometric dimensional design: the ratio of thicknesses between the piezoelectric ceramics and the metal diaphragm, and the lateral dimension of the piezoelectric ceramics length, has been obtained and based on the optimized parameters, a micropump was fabricated and tested. The tested results show that such optimized dimensions have a high pump flow rate for air up to $39 \mathrm{ml} / \mathrm{min}$ and for water up to $1.8 \mathrm{ml} / \mathrm{min}$ currently. 
It is believed that the micro-pump is very promising to go into the commercial market to handle aggressive liquids and gases at low pressures in bio-medical and drug delivery systems. But it is worthwhile to admit that there is still a lot further work to be done before this can happen. For example, there are needs to study effects of the backing pressure on the pumping rate and further to reduce the plastic body material (PEEK) cost and silicone elastomer materials (PDMS) cost. Our targeted pump costs at high volume production ( $>$ $10^{6} /$ year) is a needed control within the order of magnitude of $1 € /$ pump (plastic part: $0.1 €$, piezoelectric ceramics: $3 \times 0.1 €$, metal diaphragm: $0.02 €$, assembly and test: $0.4 €$ ).

\section{Acknowledgement}

The authors gratefully acknowledge the support from the network excellence "MultiMaterial-Micro-Manufacture" (4M). 


\section{References}

1. A. Manz, N. Graber, and H. M. Widermer, Miniaturized total chemical analysis systems: a novel concept of chemical sensing, Sensors and Actuators B, Chemicals, 1, 244-248, 1990.

2. A. Manz et al., Micromachining of monocrystalline silicon and glass for chemical analysis systems-a look into next century's technology or just a fashionable craze? Trends, Anal. Chem. 10, 144-149, 1991.

3. J. M. Smits, Piezoelectric micropump with three values working peristaltically, Sensors and Actuators A 21 to 23, 203-206, 1990.

4. H. T. G. van Lintel, F.C. M. Van de Pol, S. Bouwstra, A piezoelectric micropump based on micromachining of silicon, Sensors and Actuators A 15, 153-167, 1988.

5. N. T. Nguten et al., MEMS-micropumps: a review, Journal of Fluids Engineering, 124, 384-392, 2002.

6. F. E.H. Tray, Microfluidics and BioMEMS Applications, Boston, MA: Kluwer Academic, 2002, Chapter 1.

7. C. Yamahata et al., Plastic micropump with ferrofluidic actuation, Journal of microelectromechanical systems, 14(1), 96-102, 2005.

8. R. Linnemann, M. Richter, P. Woias, A self priming and bubble-tolerant silicon micropump for space research, Proceedings of 2nd Round Table on Micro/Nano Technologies for Space (ESTEC), Noordwijk, The Netherlands, 15-17 October 1997, 83-90.

9. D. R. Reyes, D. Iossifidis, P. A. Auroux, and A, Man, Micro total analysis systems, 1, Introduction, theory, and technology, Anal. Chemicals, 74, 2623-2636, 2002.

10. S. Bohm, W. Olthuis, P. Bergveld, A plastic micropump constructed with conventional techniques and materials, Sensors and Actuators, 77, 223-228, 1999.

11. A. Olsson, et al., Valve-less diffuser micropumps fabricated using thermoplastic replication, Sensors and Actuators, 64, 63-68, 1998. 
12. T. Merkel, M. Graeber, and L. Pagel, A new technology for fluidic microsystems based on PCB technology, Sensors and Actuators, 77, 98-105, 1999

13. Available from http://www.thinxxs.de, 2006.

14. Available from http://www.bartels-mikrotechnik.de, 2006.

15. Available from http://www.star-micronics.co.jp, 2006.

16. M. Richter et al., Development of a multi-material micropump, Journal of Mechanical Engineering Science, Part C, 220, 1619-1624, 2006.

17. M. Zhu, P. Kirby, M. Richter, Y. Congar, A. Diehl, R. Voelkl, Modelling and simulation of piezoelectric actuation and reliability of micro-pumps, second international conference on multi-material micro manufacture, 20-22 September 2006, Grenoble, France, 263- 266, 2006. 


\section{Authors’ short biography}

Meiling Zhu received her Ph. D. at the Department of Mechanical Engineering, Southeast University, Nanjing, China in 1995. She is working in the Microsystems and Nanotechnology Centre at the School of Applied Science of Cranfield University. Her interest is in the field of piezoelectric sensing and actuation, and energy harvesting, including piezoelectric motors, accelerometers, acoustic resonators and filters, micropumps, and piezoelectric energy harvesters. Her expertise is mainly in design, analysis, modelling and simulation, prototyping and applications of macro/micro sized piezoelectric devices.

Paul B. Kirby was born in Catford, London within the sound of Bow Bells. His studies concluded with the award of a PhD in Physics from the Cavendish Laboratory, University of Cambridge. Presently, he is a Reader in Microsystems at Cranfield University where his research has continued his career theme of developing processes that allow the introduction of new materials into electronic devices and sensors and actuators. Prior to his appointment at Cranfield he undertook fundamental material studies of amorphous silicon and III-V quantum well structures at industrial laboratories: IBM's Thomas J Watson Research Centre, Harvard University, and GEC's Hirst and Caswell Research Centres. His present interests include sputtering of ferroelectric materials, the development of passive component devices based on high dielectric constant materials, the fabrication of Piezoelectric MEMS devices including piezoelectric switches and frequency agile microwave devices and inertial sensors. A new recent interest is the development of an automated Patch Clamping microsystem. Presently he is undertaking a Walton Fellowship Award at the Tyndall Institute in Ireland.

Martin Wacklerle Martin Wackerle received his diploma degree in physics in 1999. He is working at the Fraunhofer Institute in Munich since 1999 as a member of the department 
micromechanics, actuators and fluidics. He is mainly involved in the development of silicon micro pumps and free jet dispensers.

Markus Herz received a diploma in mechanical engineering from the Technical University Munich, Germany and the Universidad Politécnica de Madrid, Spain in 2006. He is working as a research fellow at the Fraunhofer Institute for Reliability and Microintegration in Munich. His current fields of interest include dosing aspects of micropumps, high performance micropumps and micromechanic manufacturing.

Martin Richter Martin Richter received his diploma degree in physics in 1993. He finished his $\mathrm{PhD}$ in the field of microfludic systems in 1998. He is working at the Fraunhofer Institute in Munich, since 2001 as head of department micromechanics, actuators and fluidics. His scientific interest is in the field of microfluidic actuators like piezoelectrically and electrostatically driven micropumps made of silicon, metals or plastics, active and passive NC and NO valves, mixers, reactors, jet-spotters, flow sensors and the combination of these components to systems. 
Table 1 Material parameters and geometrical dimensions

\begin{tabular}{|c|c|}
\hline \multicolumn{2}{|l|}{ Metal diaphragm dimensions } \\
\hline Length $l(\mathrm{~mm})$ & 44 \\
\hline width $w(\mathrm{~mm})$ & 16.95 \\
\hline thickness $t(\mathrm{~mm})$ & 0.05 \\
\hline \multicolumn{2}{|l|}{ Piezoelectric plate dimensions } \\
\hline Length $l_{p}(\mathrm{~mm})$ & 9.6 \\
\hline Width $w_{p}(\mathrm{~mm})$ & 9.6 \\
\hline Thickness $t_{p}(\mathrm{~mm})$ & 0.1 \\
\hline \multicolumn{2}{|l|}{ Metal diaphragm (Argeste) } \\
\hline Young's modulus $\mathrm{E}_{\mathrm{s}}(\mathrm{GPa})$ & 200 \\
\hline Poisson ratio $\mu_{\mathrm{s}}$ & 0.3 \\
\hline Density $\left(\mathrm{kg} / \mathrm{m}^{3}\right)$ & 7800 \\
\hline \multicolumn{2}{|l|}{ Chamber } \\
\hline Area $l \times l(\mathrm{~mm} \times \mathrm{mm})$ & $14 \times 14$ \\
\hline Fillet radius $(\mathrm{mm})$ & 4.8 \\
\hline \multicolumn{2}{|c|}{$\begin{array}{l}\text { Piezoelectric material from Block Technique } \\
\text { PPK11 }\end{array}$} \\
\hline \multicolumn{2}{|c|}{ Compliance constant $10^{-12}(1 / \mathrm{Pa})$} \\
\hline \multicolumn{2}{|l|}{$s_{11}^{E}$} \\
\hline$s_{33}^{E}$ & $\begin{array}{l}15.9 \\
19.0\end{array}$ \\
\hline Density $\rho_{\mathrm{p}}\left(\mathrm{kg} / \mathrm{m}^{3}\right)$ & 8100 \\
\hline \multicolumn{2}{|c|}{ Piezoelectric constant $\left(10^{-12} \mathrm{C} / \mathrm{N}\right)$} \\
\hline$d_{33}$ & 680 \\
\hline$d_{31}$ & 350 \\
\hline \multicolumn{2}{|l|}{ Dielectric constant } \\
\hline$\varepsilon_{11 / \varepsilon_{0}}$ & 5000 \\
\hline$\varepsilon_{33 / \varepsilon_{0}}$ & 5000 \\
\hline Applied voltage (volts) & 100 \\
\hline
\end{tabular}




\section{List of Figures}

Fig. 1 Exploded view of the micro-pump

Fig. 2 Photo of the plastic pump body

Fig. 3 Downward deflection actuated by the left piezoelectric ceramics

Fig. 4 An example of actuation signals for pumping motion

Fig. 5 Geometric parameters of the modelled diaphragm

Fig. 6 Developed FEM model of the piezoelectric diaphragm with meshes

Fig. 7 Simulated deflections along the diaphragm when a voltage of 100 volts is applied to the left, middle and right piezoelectric ceramics, respectively

Fig. 8 Strokes and maximum stresses in the metal diaphragm as functions of applied voltages

Fig. 9 Strokes and the maximum stresses in the metal diaphragm as functions of piezoelectric length $l_{p}$

Fig. 10 Strokes and the maximum stresses in the metal diaphragm as functions of the ratio of $t_{p} / t$, where $t=0.05 \mathrm{~mm}$ and $t_{p}=0 \sim 0.20 \mathrm{~mm}$

Fig. 11 Maximum strains and stresses in the piezoelectric ceramic as functions of applied voltage

Fig. 12 Maximum strains and stresses in the piezoelectric ceramics as functions of $l_{p}$

Fig. 13 Maximum strains and stresses in the piezoelectric ceramics as functions of ratio of $t_{p} / t$, where $t=0.05 \mathrm{~mm}$ and $t_{p}=0 \sim 0.20 \mathrm{~mm}$

Fig. 14 Actuated strokes of the diaphragm at different applied voltage

Figs. 15 Forward and backward flow rates of the micropump with PDMS coating as function of actuation frequency (pumping air, $V_{a}=100$ volt)

Fig. 16 Forward and backward flow rates of the micropump with PDMS coating for water as functions of actuation frequency $\left(V_{a}=100\right.$ volt $)$ 


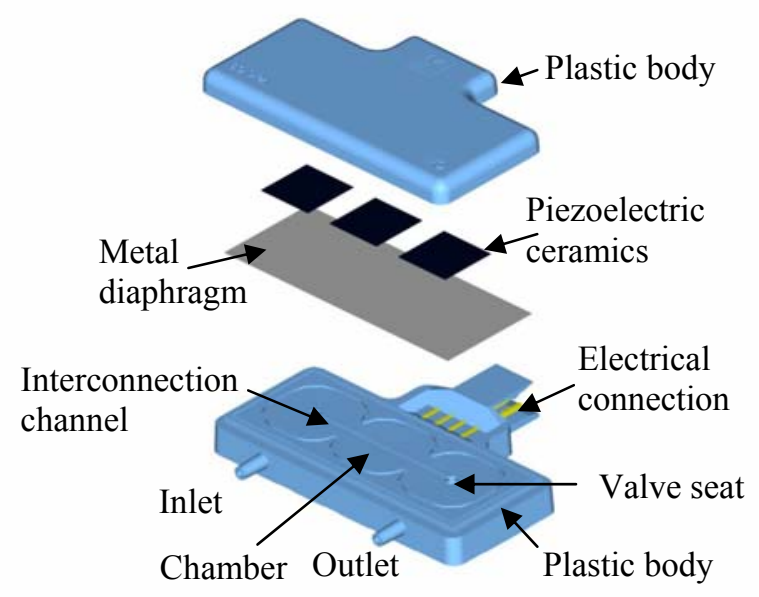

Fig. 1 Exploded view of the micro-pump 


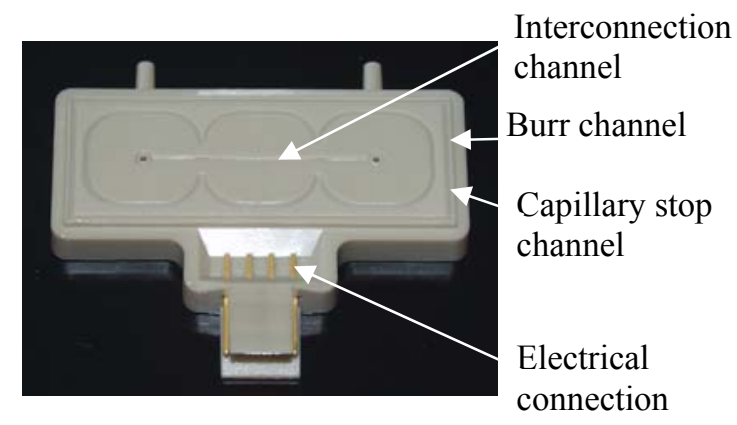

Fig. 2 Photo of the plastic pump body 


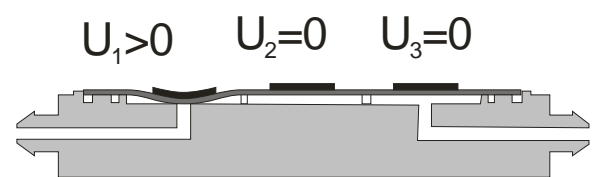

Fig. 3 Downward deflection actuated by the left piezoelectric ceramics 


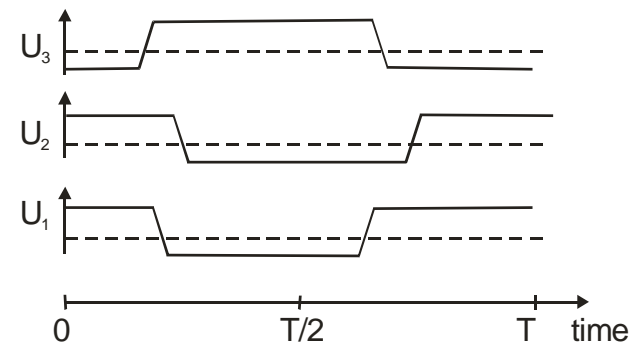

Fig. 4 An example of actuation signals for pumping motion 


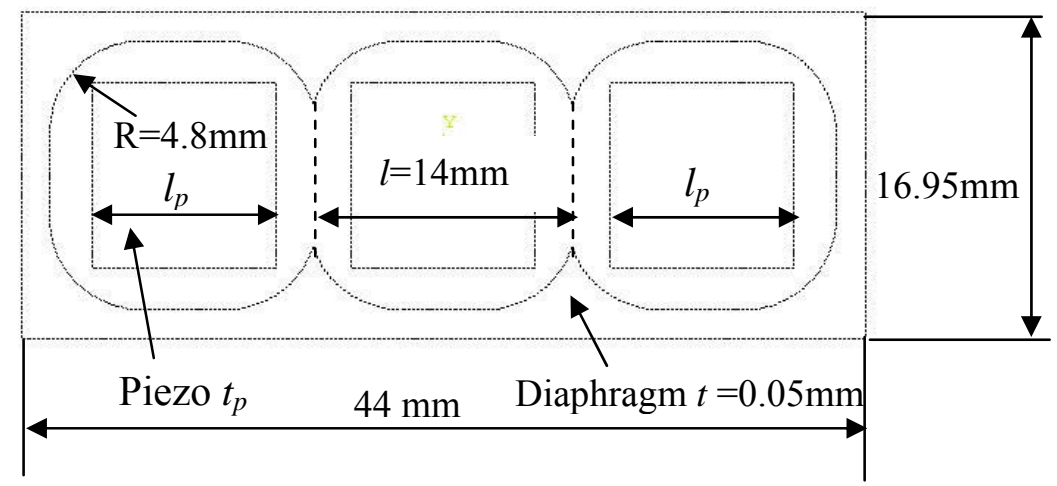

Fig. 5 Geometric parameters of the modelled diaphragm 


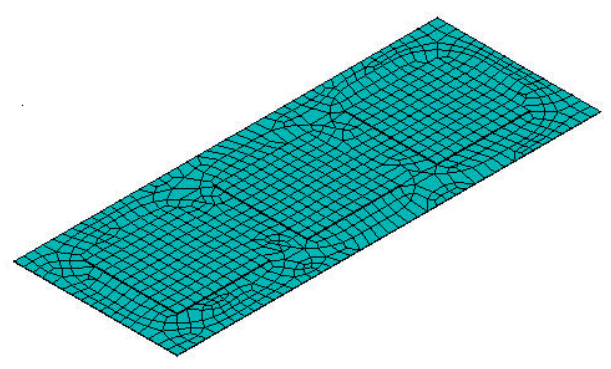

Fig. 6 Developed FEM model of the piezoelectric diaphragm with meshes 


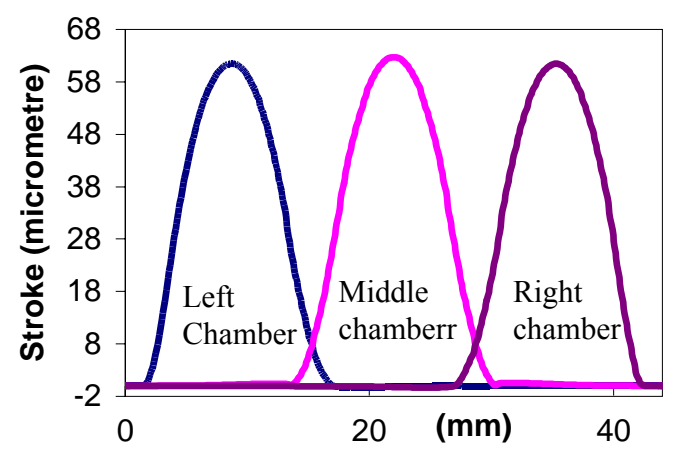

Fig. 7 Simulated deflections along the diaphragm when a voltage of 100 volts is applied to the left, middle and right piezoelectric ceramics, respectively. 


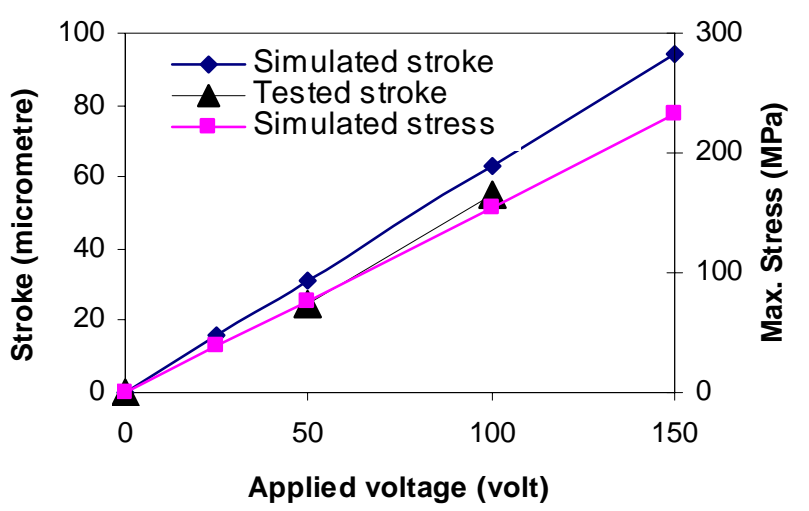

Fig. 8 Strokes and maximum stresses in the metal diaphragm as functions of applied voltages 


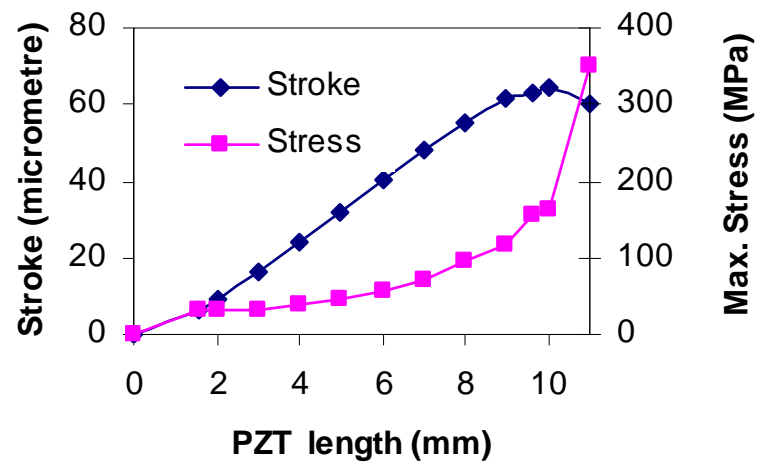

Fig. 9 Strokes and the maximum stresses in the metal diaphragm as functions of piezoelectric length $l_{p}$ 


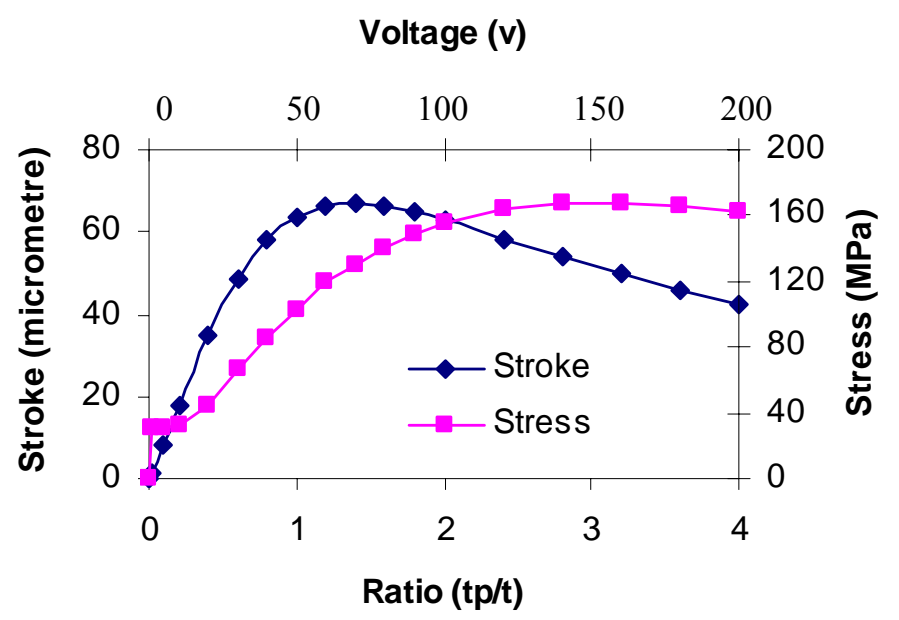

Fig. 10 Strokes and the maximum stresses in the metal diaphragm as functions of the ratio of $t_{p} / t$, where $t=0.05 \mathrm{~mm}$ and $t_{p}=0 \sim 0.20 \mathrm{~mm}$ 


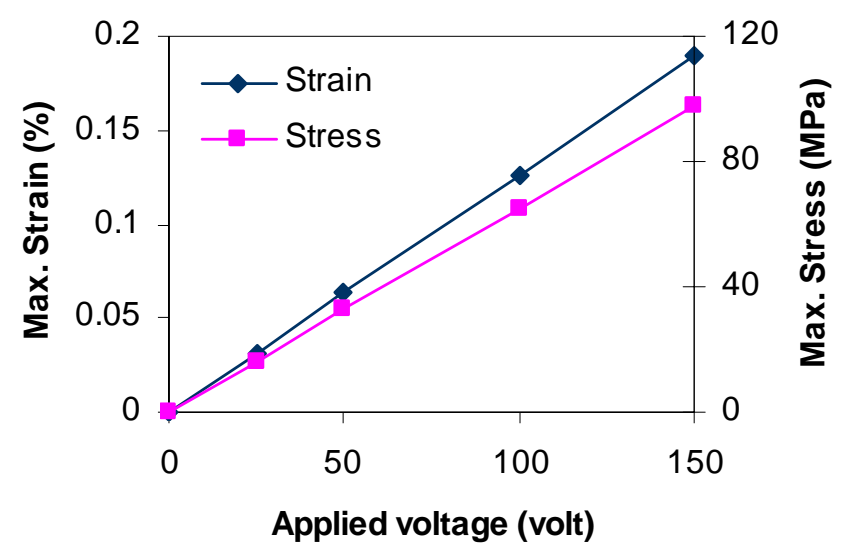

Fig. 11 Maximum strains and stresses in the piezoelectric ceramic as functions of applied voltage 


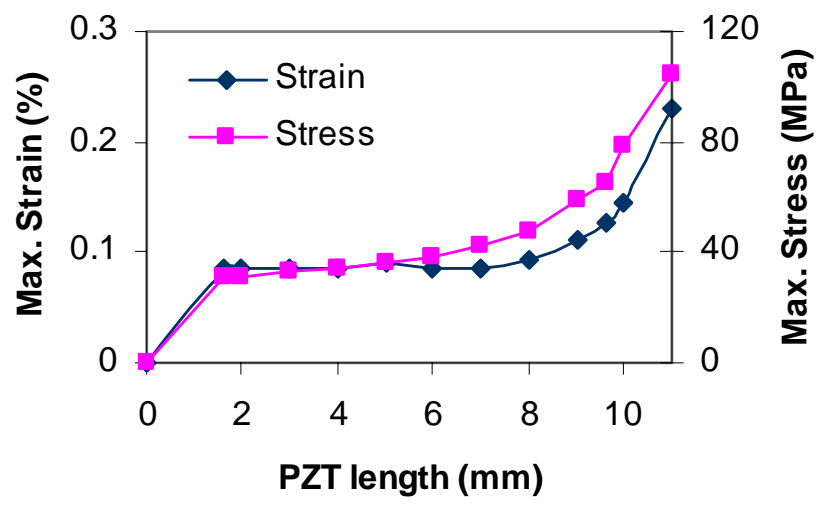

Fig. 12 Maximum strains and stresses in the piezoelectric ceramics as functions of $l_{p}$ 


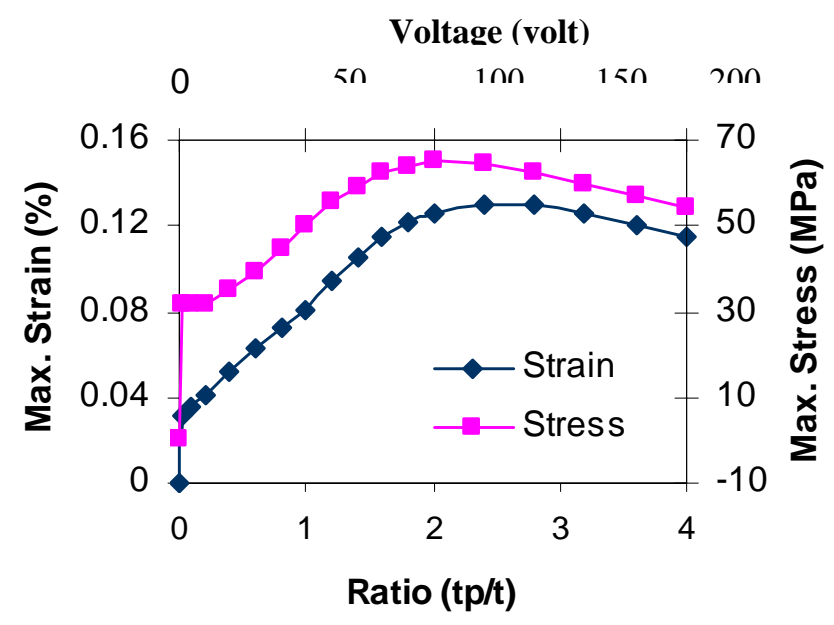

Fig. 13 Maximum strains and stresses in the piezoelectric ceramics as functions of ratio of $t_{p} / t$, where $t=0.05 \mathrm{~mm}$ and $t_{p}=0 \sim 0.20 \mathrm{~mm}$ 


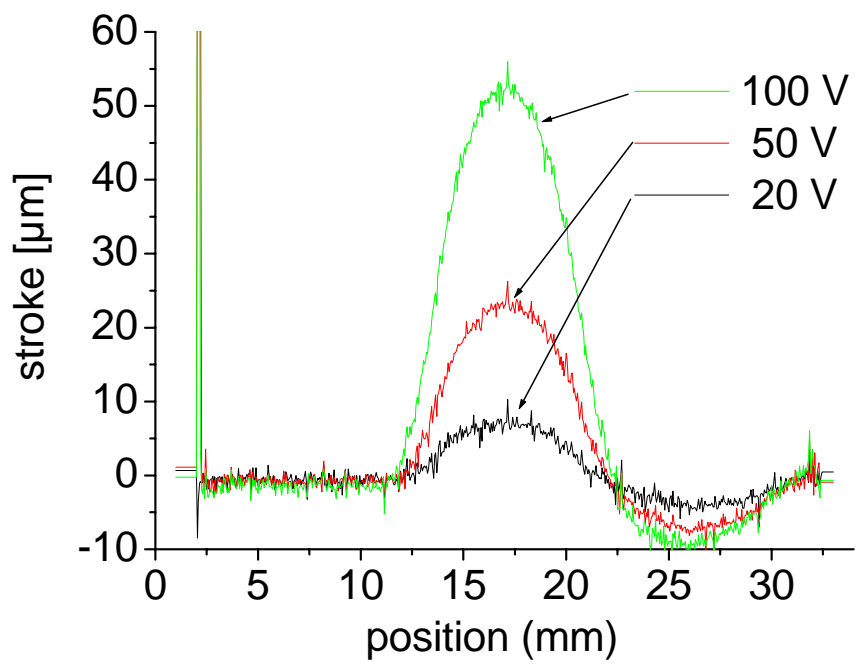

Fig. 14 Actuated strokes of the diaphragm at different applied voltage 


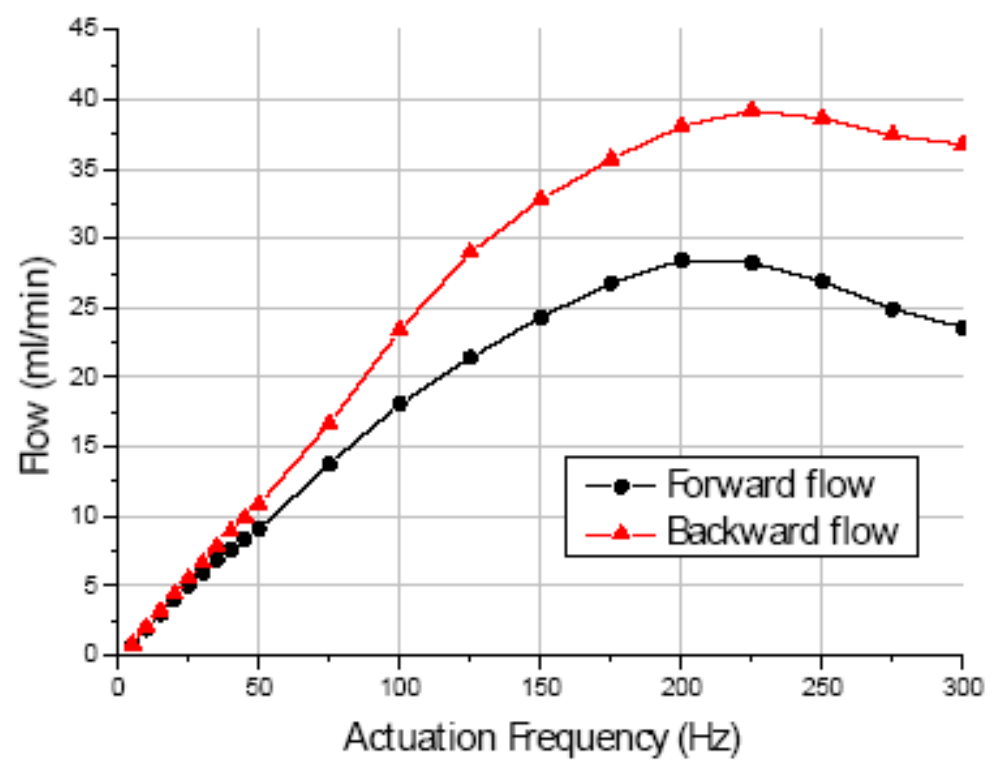

Figs. 15 Forward and backward flow rates of the micropump with PDMS coating as function of actuation frequency (pumping air, $V_{a}=100$ volt ) 


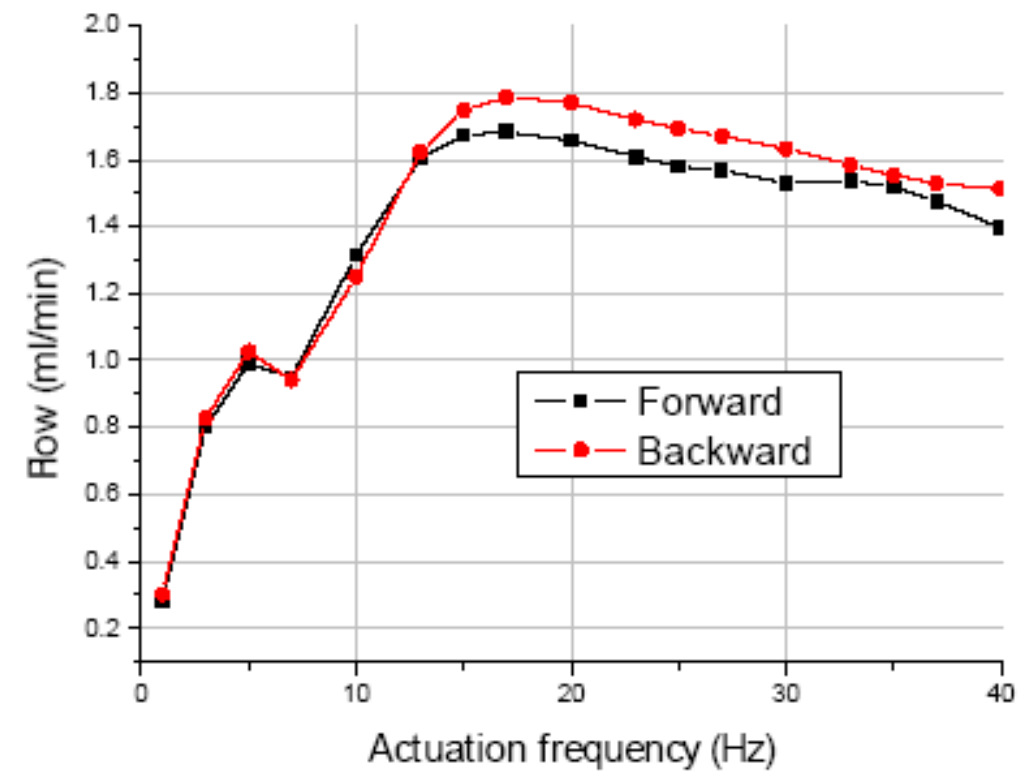

Fig. 16 Forward and backward flow rates of the micropump with PDMS coating for water as functions of actuation frequency $\left(V_{a}=100\right.$ volt $)$ 